\title{
Energy efficiency of compressed spectrum sensing in wideband cognitive radio networks
}

\author{
Qi Zhao ${ }^{1 *}$, Zhijie $\mathrm{Wu}^{2}$ and Xiaochun $\mathrm{Li}^{1}$
}

\begin{abstract}
In cognitive radio networks, wideband spectrum sensing (WSS) has been advocated as an effective approach to increase the spectrum access opportunity of the secondary users. On the other hand, because of the increase of sampling rate and sensing time duration cost by the analog-to-digital converter (ADC), energy saving has been a significant problem for WSS. In this paper, taking advantage of the frequency-domain sparsity of the wideband spectrum, a WSS scheme combining compressed sensing and multi-band joint detection technique is proposed to reduce the energy consumption. Based on extensive analysis and simulation, we identify the sparsity order of the wideband spectrum, the received signal-to-noise ratio (SNR) of the primary signal, and the compression rate employed in sampling as three key factors that affect the sensing performance. In particular, we derive a closedform analytical model of the scheme. Based on these observations, the energy efficiency, defined as the ratio of the spectrum access opportunity to the energy consumption, is maximized through the optimization process of the compression rate under the sensing performance constraints. We also indicate the uniqueness of the optimal compression rate for maximizing the energy efficiency. Numerical and simulation results show that our proposed scheme is more energy efficient if the wideband spectrum is sparser in the frequency domain.
\end{abstract}

Keywords: Cognitive radio network, Distributed cooperation, Spectrum allocation, Random broadcast

\section{Introduction}

In cognitive radio networks (CRNs), the secondary users (SUs) can opportunistically access the spectrum bands unoccupied by the primary users (PUs). As a key technology in CRNs, spectrum sensing is widely used by SUs to periodically detect the spectrum bands. It is well known that the greater the bandwidth of the spectrum being detected, the more opportunity for SUs to access. Therefore, wideband spectrum sensing (WSS) $[1,2]$ is attracting attention as an effective approach for detecting continuous multiple bands simultaneously. Since WSS can be easily performed in local sensing, i.e., it can be implemented by any SU independently, the communication overhead for network coordination can be greatly reduced. Hence, WSS is suitable for large-scale CRNs with quite a lot of SUs.

\footnotetext{
* Correspondence: zhaoqi@buaa.edu.cn

${ }^{1}$ School of Electronic Information Engineering, Beihang University, Beijing 100191, China

Full list of author information is available at the end of the article
}

On the other hand, the increasing energy consumption has been a significant problem for WSS [1,3]. The energy consumption in the spectrum sensing is mainly caused by the analog-to-digital converter (ADC), which is proportional to the sensing time duration and the sampling rate [1]. However, because of the wide range of the spectrum bandwidth for WSS, long sensing time duration and high sampling rate are always needed by the ADC. There are two kinds of the traditional WSS schemes, the sequential sensing scheme $[1,3]$ and the parallel multi-band detection scheme [4]. Originally, constrained by the low sampling rate of ADCs, SU divides the wideband spectrum into multiple sub-bands and then detects them successively, namely the sequential sensing scheme. As the high-speed sampling technique develops, SU can directly sample the wideband spectrum using a high-speed ADC and then detect all the sub-bands simultaneously, namely the parallel multiband detection scheme. Compared with the sequential sensing scheme, the structure of the parallel multi-band

\section{实 Springer}

(c) 2016 Zhao et al. Open Access This article is distributed under the terms of the Creative Commons Attribution 4.0 International License (http://creativecommons.org/licenses/by/4.0/), which permits unrestricted use, distribution, and reproduction in any medium, provided you give appropriate credit to the original author(s) and the source, provide a link to the Creative Commons license, and indicate if changes were made. 
detection scheme is more convenient for WSS. Furthermore, the parallel multi-band detection scheme is more energy efficient by improving the sampling rate to shorten the sensing time duration.

In practice, the wideband spectrum is divided into several non-overlapping narrowband channels assigned to different PUs, and the emergence of different PUs' signal is entirely independent. Therefore, only a minority of channels are occupied by PUs at the same time, namely that the wideband spectrum is sparser in the frequency range. Taking advantage of such sparsity of wideband spectrum, compressed sensing (CS) has recently been proposed to reduce the sampling rate below the Nyquist rate [5-11]. For this reason, the CS-based spectrum sensing methods have been proposed as an efficient approach for energy saving in WSS.

Tian and Giannakis introduced two kinds of compressed sensing methods (called multi-step and onestep) to recover the signal by wavelet approach [5] and then developed the signal recovery idea and performed the distributed compressive spectrum sensing in the cooperative multi-hop CRNs [6]. However, for spectrum sensing and detection, accurately reconstructing the original signal is unnecessary. Following this idea, Polo et al. presented a compressive wideband spectrum sensing scheme by sampling the analog signal using analog-to-information convert and then focused on the performance of edge spectrum and power spectrum density recovery [7]. The authors in $[8,9]$ also suggested to only reconstruct the signal's power spectrum, in order to optimize the sensing performance at the expected probabilities of detection and false alarm. As well known, the final purpose of spectrum sensing is to find the spectrum access opportunities for SUs $[1,5]$. Based on this point, all the aforementioned methods, aiming at the signal reconstruction performance in $[5,6]$ or the sensing performance in [7-9], give no considerations to the optimization problem of the spectrum access opportunity. In this paper, we have a further study on this problem, combining the challenge of energy consumption in WSS. We propose a reconstruction structure aiming at optimizing the energy efficiency, which is defined as the ratio of SU's spectrum access opportunity to the energy consumption.

The proposed design includes (i) a sensing scheme combining the compressed sensing and the multi-band joint detection, (ii) the establishment of an analytical model of the scheme, and (iii) the optimization of the compression rate for energy efficiency maximization under the sensing performance constraints. The contributions of this paper can be generalized as follows.

In comparison with the traditional WSS schemes, the proposed scheme reduces the sampling rate at the subNyquist rate and takes a shorter sensing time duration, both of which are greatly favorable to energy saving. Numerical and simulation results show that the performance of energy saving is better if the wideband spectrum is sparser in the frequency domain.

Although the compressed sensing has been suggested to reduce the sampling rate in WSS, the energy efficiency and the optimization of compression rate have never been considered. However, our study indicates the uniqueness of the optimal compression rate for maximizing the energy efficiency.

Furthermore, lots of relevant studies are developed under the ideal conditions. As in $[10,11]$, the authors deduced the minimum required number of measurements for sparsity estimation and signal reconstruction, respectively, but in the noise-free case. Based on extensive analysis and simulation, we identify the sparsity order, the received signal-to-noise ratio (SNR), and the compression rate as three key factors when performing our proposed scheme and then quantify these contributions by mathematical approximation to support the theoretical derivation.

In addition, a joint SNR and sparsity order estimation, and the conditions for implementing the compressed sensing, are suggested to give consideration to the practical application of the proposed scheme.

The remainder of this paper is organized as follows. Section 2 describes the signal and network model. Section 3 presents the composition of the proposed scheme, formulates the energy efficiency maximization problem, and derives the optimal compression rate. Section 4 shows the performance analysis and simulations. Finally, Section 5 concludes the paper.

\section{Network mode}

Consider a wideband spectrum that is divided into $K$ non-overlapping sub-bands. During a constant sensing time duration, we model the detection problem on the sub-band $k$ as one choosing between a hypothesis $H_{0, k}$, which represents the absence of the primary signals, and an alternative hypothesis $H_{1, k}$, which represents the presence of the primary signals, $k \in\{1,2, \ldots, K\}$. Moreover, we have $\operatorname{Pr}\left(H_{0, k}\right)+\operatorname{Pr}\left(H_{1, k}\right)=1$, where $\operatorname{Pr}(\cdot)$ is the probability of occurrence. Without loss of generality, we assume that $\operatorname{Pr}\left(H_{0, k}\right)=\operatorname{Pr}\left(H_{0}\right)$, and $\operatorname{Pr}\left(H_{1, k}\right)=\operatorname{Pr}$ $\left(H_{1}\right), \forall k$. Let $S_{k}(t)$ and $H_{k}(t)$ be the primary signal and the coefficient of the sub-band $k$ at any given time $t$, respectively. Then, define $X(t)=\left\{X_{1}(t), X_{2}(t), \cdots, X_{K}(t)\right\}$, where $X_{k}(t)=H_{k}(t) S_{k}(t)$. Thus, the received signals at SUs can be represented as $R(t)=X(t) \cdot 1(k, t)+V(t)$, where $V(t)$ is the additive white Gaussian noise and $1(k, t)$ is a $K \times 1$ column vector in which the $k$ th element is equal to 1 in the hypothesis $H_{1, k}$ and is equal to 0 in the hypothesis $H_{0, k}$. In addition, we define the received SNR 
on the sub-band $k$ as $\gamma_{k}=p_{k} / p_{0}$, where $p_{k}$ is the received power of the primary signals and $p_{0}$ the noise power.

\section{Proposed wideband spectrum sensing scheme}

\subsection{Scheme description}

Figure 1 illustrates our proposed scheme, which is tailored to the WSS. The scheme includes five modules: the compressed sensing module $\left(\mathbf{A}_{\mathbf{1}}\right)$, the multi-band joint detection module $\left(\mathbf{A}_{\mathbf{2}}\right)$, the energy efficiency maximization module $\left(\mathbf{A}_{3}\right)$, the sparsity order and SNR estimation module $\left(\mathbf{B}_{1}\right)$, and the compressed sampling decision module $\left(\mathbf{C}_{\mathbf{1}}\right)$. The work flow of Fig. 1 can be described as follows.

If $\mathbf{C}_{\mathbf{1}}$ is satisfied, i.e., the spectrum is sparse and all the elements of $\gamma=\left\{\gamma_{k}, \forall k\right\}$ are above the lower limit $\bar{\gamma}$, in $\mathbf{A}_{1}, R(t)$ is compressed sampled at the optimal sub-Nyquist sampling rate, given by $\mathbf{A}_{3}$, for the energy efficiency maximization, and then reconstructed through the orthogonal matching pursuit (OMP) method [4] as $\hat{\boldsymbol{R}}=\{\hat{R}(n)\}, n=\{1,2, \ldots, N\}$. Otherwise, $a \cdot \exp (b \cdot \delta)+c \cdot \exp (d \cdot \delta)$ is sampled at the Nyquist rate as $\boldsymbol{R}=\{R(n)\}, n=\{1,2, \ldots, N\}$. Next, $\hat{\boldsymbol{R}}$ (or $\boldsymbol{R}$ ) is used by $\mathbf{A}_{2}$ for the parallel multi-band detection. Meanwhile, the summary statistic of each sub-band $k$, $\hat{T}_{k}$ (or $T_{k}$ ), is used by $\mathbf{B}_{\mathbf{1}}$ to estimate the sparsity order and $\gamma$.

As an example of normal ADC chips, the energy consumption of AD9257 can be described as [12]

$$
E\left(f_{s}, \tau_{s}\right)=\left(2.33 f_{s}+151.67\right) \cdot \tau_{s},
$$

where $f_{s}$ is the sampling rate and $\tau_{s}$ is the sampling time duration; the units of $E, f_{s}$, and $\tau_{s}$ are $\mathrm{mW}, \mathrm{Mbps}$, and $\mathrm{s}$, respectively. It can be observed that when $f_{s}$ is in a relatively low regime, i.e., $f_{s} \leq 65 \mathrm{MHz}, E$ is mainly dependent on $\tau_{s}$. This indicates that for a given $N$, where $N=f_{s} \tau_{s}$, increasing $f_{s}$ (as a means of shortening $\tau_{s}$ ) is efficient to save energy. Compared with the sequential sensing scheme, the parallel detection structure of $\mathbf{A}_{\mathbf{2}}$ is more suitable for sampling the wideband spectrum at a high $f_{s}$. However, when $f_{s}>65 \mathrm{MHz}$, the energy consumption caused by the increase of $f_{s}$ is apparent. To overcome this, $\mathbf{A}_{\mathbf{1}}$ is applied to reduce $f_{s}$ to a level of less than the Nyquist rate for further energy saving.

\subsection{Compressed sensing $\left(A_{1}\right)$}

In most cases, since $X(t)$ does not occupy all the $K$ subbands, it may be sparse in the frequency domain. According to the compressed sensing theory [13], if $\boldsymbol{X}(t)$ is truly sparse, it can be sampled at the sub-Nyquist rate and then reconstructed with slight errors. Denote the sparsity order of $\boldsymbol{X}(t)$ as $\rho=\frac{\text { the number of occupied sub-bands }}{K}$ and the compression rate for sampling as $\delta=f_{\mathrm{cs}} / f_{N}$, $\delta \in(0,1)$, where $f_{\text {cs }}$ is the compressed sampling rate and $f_{N}$ is the Nyquist rate. It is well known that $\delta$ must be larger than $\rho$ in order to reconstruct the sparse signals. Moreover, the reconstruction performance is improved with the increase of $\delta$.

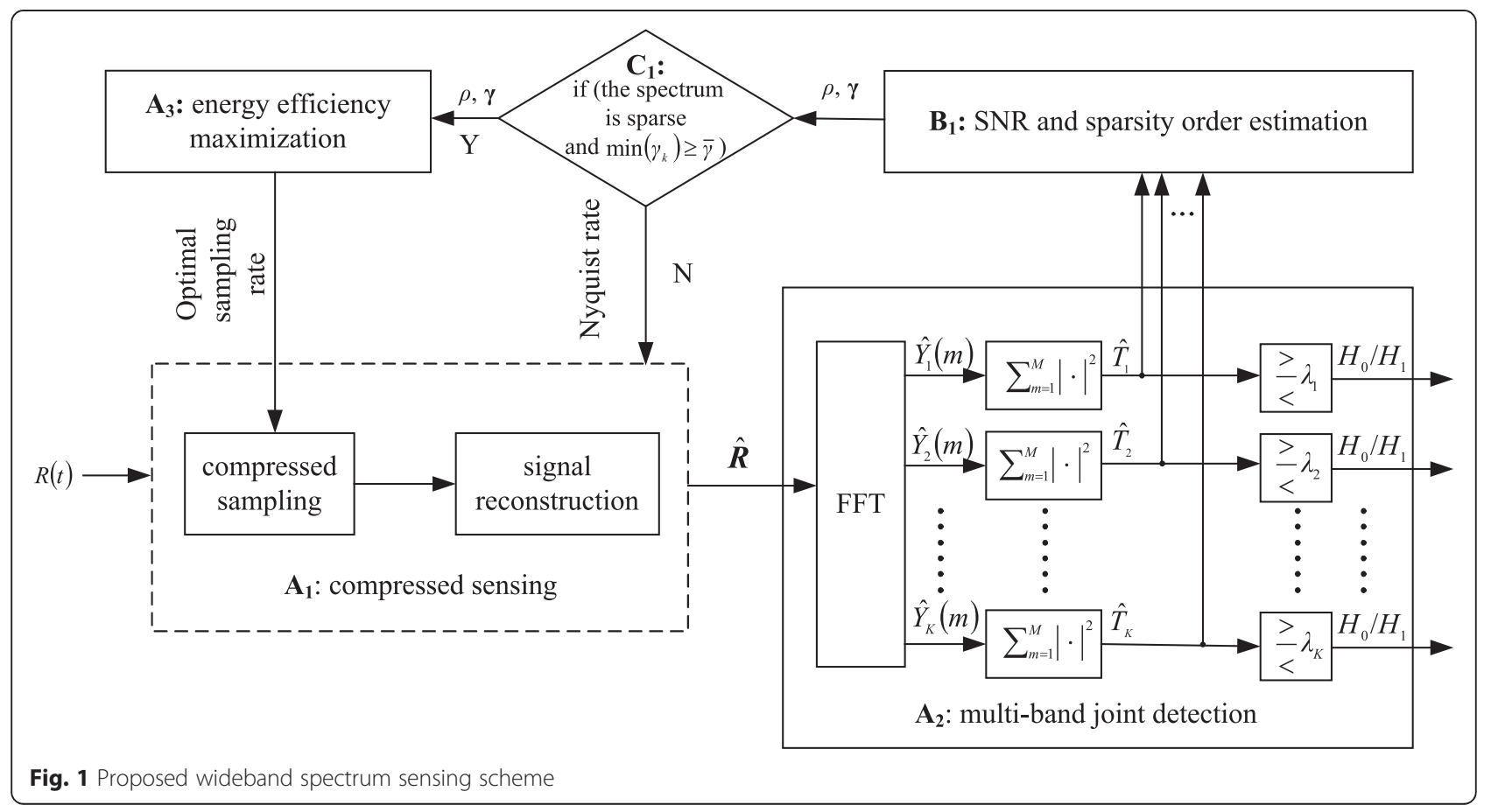


Module $\mathbf{A}_{\mathbf{1}}$ consists of two sequential sub-modules, the compressed sampling and the signal reconstruction. It has been shown that samples acquired from Gaussian and Bernoulli random sampling matrices have a better chance to perfectly recover sparse signals than other types of sampling matrices [6]. There are several options for hardware implementations of compressed sampling. An exemplary sampler is called an analog-to-information converter (AIC). It has a serial concatenated structure with three main components: a wideband modulator made of a pseudo-random chipping sequence, a low-pass filter, and a low-rate conventional ADC $[10,11]$. In this paper, for a given $1 \times N$ vector $\boldsymbol{R}$, we simulate the process of compressed sampling as follows:

- Choose an $N \times N$ discrete Fourier transform (DFT) matrix as the sparse representation basis $\boldsymbol{\Psi}$, then map $\boldsymbol{R}$ into the sparse domain as $\boldsymbol{\Theta}=\boldsymbol{\Psi}^{\mathrm{T}} \boldsymbol{R}^{\mathrm{T}}$.

- Choose a $V \times N$ uniform Gaussian random sampling matrix $\boldsymbol{\Phi}$, where $V=\lceil\delta N\urcorner$ ( $\lceil x\rceil$ is the smallest integer not less than $x$ ), then calculate the measurement matrix as $\boldsymbol{W}=\boldsymbol{\Phi} \boldsymbol{\Psi}^{\mathrm{T}} \boldsymbol{R}^{\mathrm{T}}$.

Next, we represent $\Phi \Psi^{\mathrm{T}}$ as $\Lambda$ and feed $\Lambda$ and $W$ into the OMP method to calculate $\hat{\boldsymbol{R}}$, i.e., the reconstruction of $\boldsymbol{R}$. OMP is a kind of greedy iterative algorithm for reconstruction. In each iteration, the column of $\Lambda$ having the highest correlation with the error vector is selected and is used to update the approximate solution of $\boldsymbol{R}$. The iterative process will not be stopped until the error is small enough or the number of iterations is equal to $V$. In detail, the process of signal reconstruction through OMP method can be described as follows:

- Initialization. Denote the iteration index as $i=0$, the error vector as $\boldsymbol{r}^{(0)}=\boldsymbol{W}$, and the expected nonnegative error vector as $\overline{\boldsymbol{r}}$. Let $\hat{\boldsymbol{R}}^{(0)}=0, \boldsymbol{\Omega}^{(0)}=\varnothing$, where 0 is a $1 \times N$ vector consisting of zero elements.

- Step 1: Calculate the inner products of the error vector and the columns of $\boldsymbol{\Lambda}, \boldsymbol{g}^{(i)}=\boldsymbol{\Lambda}^{\mathrm{T}} \boldsymbol{r}^{(i-1)}$.

- Step 2: Find the maximum element of $\left|\boldsymbol{g}^{(i)}\right|$, then get its column index as $j=\underset{n \in\{1,2, \cdots, N\}}{\arg \max }\left|\boldsymbol{g}^{(i)}(n)\right|$.

- Step 3: Update $\boldsymbol{\Omega}^{(i)}$ as $\boldsymbol{\Omega}^{(i-1)} \cup\{\boldsymbol{\Lambda}(:, j)\}$, where $\boldsymbol{\Lambda}(:, j)$ represents all the elements of the jth column of $\boldsymbol{\Lambda}$.

- Step 4: Calculate the approximate solution of $\boldsymbol{R}$ through the least square method, $\hat{\boldsymbol{R}}^{(i)}=$ $\left(\left(\boldsymbol{\Omega}^{(i)}\right)^{\mathrm{T}} \boldsymbol{\Omega}^{(i)}\right)^{-1}\left(\boldsymbol{\Omega}^{(i)}\right)^{\mathrm{T}} \boldsymbol{W}$.
- Step 5: Update $\boldsymbol{r}^{(i)}$ as $\boldsymbol{W}-\boldsymbol{\Lambda} \hat{\boldsymbol{R}}^{(i)}$.

- Step 6: If $\left|\boldsymbol{r}^{(i)}\right| \leq \overline{\boldsymbol{r}}$ or $i=V$, output $\hat{\boldsymbol{R}}=\hat{\boldsymbol{R}}^{(i)}$ and stop. Otherwise, go to step 1.

So far, the compressed sampling and the signal reconstruction sub-modules have been present, and the simulation model of the compressed sensing can be established as a result.

\subsection{Multi-band joint detection $\left(A_{2}\right)$}

To facilitate the description, we introduce the multiband joint detection module when sampling at $f_{N}$. The module implements the WSS in three steps:

- Calculate the discrete frequency response of $\boldsymbol{R}$ (denoted as $\boldsymbol{Y}=\{Y(n)\}, n=\{1,2, \ldots, N\}$ ) through an $N$-point fast Fourier transform (FFT).

- For each sub-band $k$, calculate summary statistic $T_{k}$ as the sum of the received signal energy over an interval of $M$ samples, i.e., $T_{k}=\sum_{m=1}^{M}\left|Y_{k}(m)\right|^{2}$, where $M=N / 2 K$ and $n=(k-1) M+m$.

- Compare $T_{k}$ with the threshold $\lambda_{k}$ to make a decision whether the primary signal is present or not.

According to [4], for a large $M$, the probability distribution functions (PDFs) of $T_{k}$ are close to normal distributions and can be represented as $N\left(\mu_{i, k}, \sigma_{i, k}^{2}\right)$, $i \in\{0,1\}$, where

$$
\mathrm{H}_{0, k}:\left\{\begin{array}{l}
\mu_{0, k}=M p_{0} \\
\sigma_{0, k}^{2}=2 M p_{0}^{2}
\end{array}, \quad \mathrm{H}_{1, k}:\left\{\begin{array}{l}
\mu_{1, k}=M\left(\gamma_{k}+1\right) p_{0} \\
\sigma_{1, k}^{2}=2 M\left(2 \gamma_{k}+1\right) p_{0}^{2}
\end{array}\right.\right.
$$

\subsection{Impact of sparsity order, compression rate, and SNR} Now, we consider the multi-band joint detection when the compressed sensing module $\mathbf{A}_{\mathbf{1}}$ is valid. The spectrum can be reconstructed through the OMP method but at the expense of causing recovery errors. The performance of signal reconstruction depends on the sparsity order and the compression rate. Thus, it is foreseeable that the two factors should also affect the detection accuracy. On the other hand, since the energy detector used at each branch of $\mathbf{A}_{\mathbf{2}}$ cannot extract the primary signal from the noise, the received SNR is the most important factor for signal detection. In what follows, we thus investigate the impact of sparsity order, compression rate, and SNR on the sensing performance of the proposed scheme.

Assume $N=512, p_{0}=-95.2 \mathrm{dBm}$, and a sparse wideband spectrum contains $10 \mathrm{TV}$ bands each with $6-\mathrm{MHz}$ bandwidth. Within the range of $\delta \in(0,1)$, Figs. 2a and 3a 


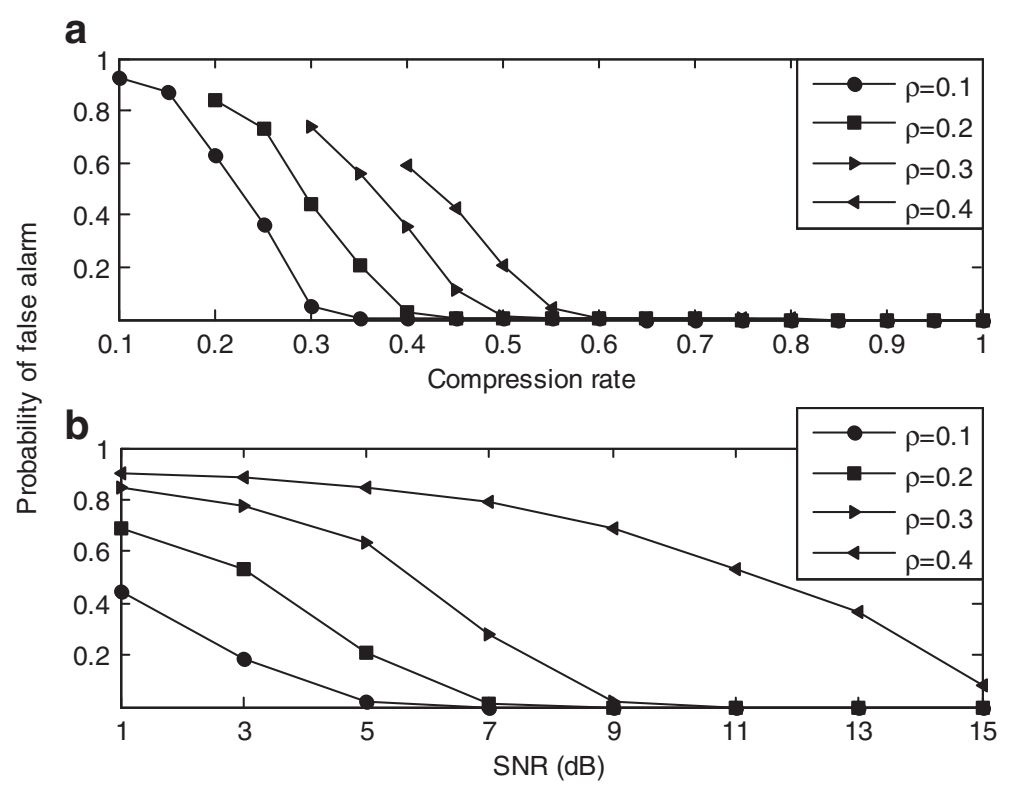

Fig. 2 Impact of sparsity order, compression rate, and SNR. a The probability of false alarm versus $\delta$, when $\gamma_{k}=9 \mathrm{~dB}$. $\mathbf{b}$ The probability of false alarm for different pairs of $\left(\rho, \gamma_{k}\right)$ as $\delta=0.5$

demonstrate that the probability of false alarm and the probability of detection rapidly drop to 0 and raise to 1 , respectively, for $\forall \rho \in\{0.1,02,0.3,0.4\}$ as the increase of $\delta$. This proves the feasibility of the scheme, i.e., the structure of the combined $\mathbf{A}_{\mathbf{1}}$ and $\mathbf{A}_{\mathbf{2}}$ is the ability to accomplish the task of WSS if the spectrum is truly sparse. In addition, for the same sensing performance, the needed compression rate becomes lower as the sparsity order decreases. Figures $2 \mathrm{~b}$ and $3 \mathrm{~b}$ show the probability of false alarm and the probability of detection, respectively, for different pairs of $\left(\rho, \gamma_{k}\right)$ as $\delta=0.5$. We can see that for any sub-band in the wideband spectrum, the detection accuracy of the status (occupied or not) is proportional to the received SNR of the primary signal. This indicates when the sparsity order and compression rate have been determined

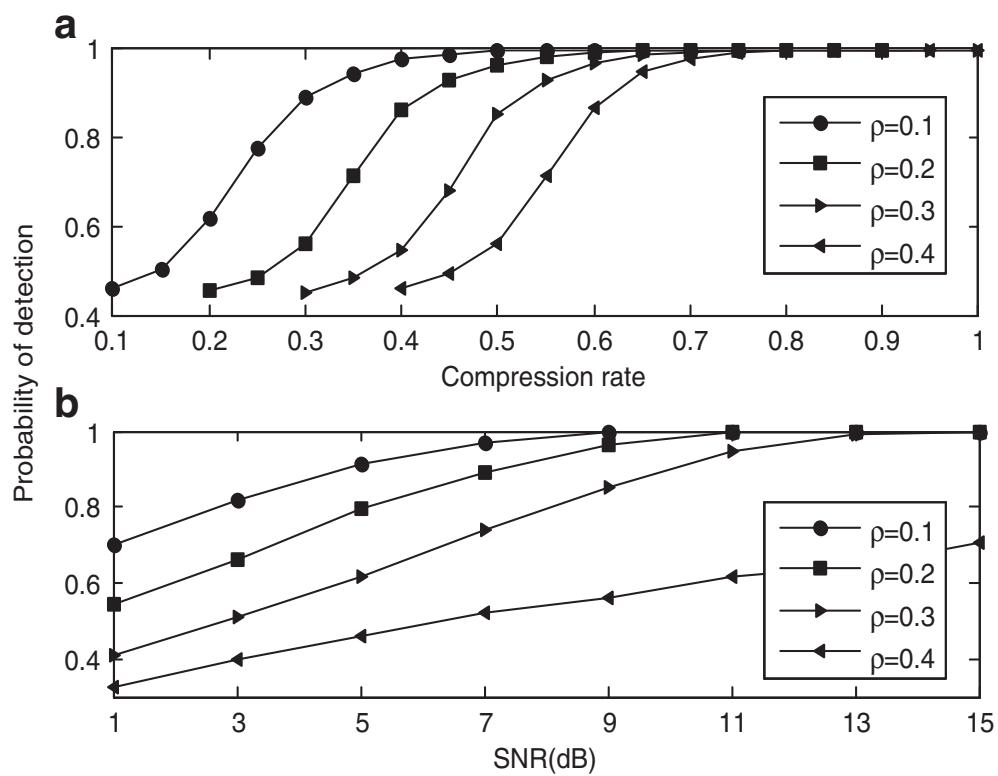

Fig. 3 Impact of sparsity order, compression rate, and SNR. a The probability of detection versus $\delta$, when $\gamma_{k}=9 \mathrm{~dB}$. $\mathbf{b}$ The probability of detection for different pairs of $\left(\rho, \gamma_{k}\right)$ as $\delta=0.5$ 
that the higher the received SNR, the better the sensing performance.

\subsection{Analysis model}

Denote that the primary signal does not occupy channel $k$ as hypothesis $H_{0, k}$, otherwise as $H_{1, k}$. Using the Central Limit Theorem (CLT) as in [4], we have that $\hat{T}_{k} \sim N$ $\left(\hat{\mu}_{i, k}, \hat{\sigma}_{i, k}^{2}\right)$, where $\hat{\mu}_{i, k}$ and $\hat{\sigma}_{i, k}^{2}$ is the mean and variance of the received primary signal, $i \in\{0,1\}$, respectively. Thus,

$$
\operatorname{Pr}\left(\hat{T}_{k}>\lambda_{k} \mid H_{i, k}\right)=Q\left(\frac{\left(\lambda_{k}-\hat{\mu}_{i, k}\right)}{\hat{\sigma}_{i, k}}\right)
$$

can be used to unify the expressions of the probability of false alarm $P_{f}^{(k)}$ (when $i=0$ ) and the probability of detection $P_{d}^{(k)} \quad($ when $i=1)$, where $Q(x)=$ $(1 / \sqrt{2 \pi}) \cdot \int_{x}^{\infty} \exp \left(-t^{2} / 2\right) d t$. Based on the extensive analysis and simulation above, we identify $\rho, \gamma_{k}$, and $\delta$ as three key factors that affect the sensing performance of the proposed scheme. Therefore, to establish a relationship between $\hat{T}_{k}$ and $T_{k}$, we denote

$$
\left\{\begin{array}{l}
\hat{\mu}_{i, k}=\alpha_{i, k}\left(\rho, \gamma_{k}, \delta\right) \cdot \mu_{i, k} \\
\hat{\sigma}_{i, k}^{2}=\beta_{i, k}^{2}\left(\rho, \gamma_{k}, \delta\right) \cdot \sigma_{i, k}^{2}
\end{array}, i \in\{0,1\}, \forall k\right.
$$

where $\alpha_{i, k}$ and $\beta_{i, k}^{2}$ are normalized coefficients, both of which are closely linked to $\rho, \gamma_{k}$, and $\delta$. Substitute Eqs. (2) and (4) into Eq. (3). For a target probability of detection $\bar{P}_{d}$, the probability of false alarm can be calculated by

$$
\begin{aligned}
P_{f}^{(k)}= & Q\left(\frac{\beta_{1, k}}{\beta_{0, k}} \sqrt{2 \gamma_{k}+1} Q^{-1}\left(\bar{P}_{d}\right)\right. \\
& \left.+\frac{\alpha_{1, k}\left(\gamma_{k}+1\right)-\alpha_{0, k}}{\sqrt{2} \beta_{0}} \sqrt{M}\right)
\end{aligned}
$$

On the other hand, for a target probability of false alarm $\bar{P}_{f}$, the probability of detection is

$$
\begin{aligned}
P_{d}^{(k)}= & Q\left(\frac { 1 } { \sqrt { 2 \gamma _ { k } + 1 } } \left(\frac{\beta_{0, k}}{\beta_{1, k}} Q^{-1}\left(\bar{P}_{f}\right)\right.\right. \\
& \left.\left.-\frac{\alpha_{1, k}\left(\gamma_{k}+1\right)-\alpha_{0, k}}{\sqrt{2} \beta_{1}} \sqrt{M}\right)\right)
\end{aligned}
$$

For simplification, we rewrite $\alpha_{i, k}$ as $\alpha_{i}$ and $\beta_{i, k}$ as $\beta_{i}$ in the following sections. The analytical expressions of the coefficients $\alpha_{i}$ and $\beta_{i}$ can be obtained from simulation results through the curve-fitting method. Since there are similar fitting results for different pairs of $\left(\rho, \gamma_{k}\right)$, we choose $\rho=0.2$ and $\gamma_{k}=9 \mathrm{~dB}$ for demonstrations. Table 1 gives the experimental data of the coefficients. It can be found that $\alpha_{1}$ converges to 1 rapidly as $\delta$ increases. Then, $\alpha_{0}$ and $\beta_{i}$ can be accurately fitted into functions of $\delta$ as

$$
a \cdot \exp (b \cdot \delta)+c \cdot \exp (d \cdot \delta)
$$

Furthermore, as shown in Table 2, $a$ is always positive while $b$ is negative. As for $c$ and $d$, at least one of them is close to zero.

\subsection{Energy efficiency maximization $\left(A_{3}\right)$}

SU's final goal is to access the spectrum and transmit its own data $[1,5]$. There are two different spectrum access schemes, namely the underlay and overlay approaches $[1,4,14]$. Under the underlay approach, SUs are allowed to transmit on occupied sub-bands but suffer to severe transmitting power limit for avoiding interference with the primary signals. On the contrary, although only the unoccupied sub-bands can be utilized when the overlay approach is selected, SUs can greatly increase their transmitting powers to improve the quality of service (QoS). Generally speaking, the overlay approach is more suitable for the communication of CRN; thus, it is considered in this paper and the spectrum access opportunity of SU can be represented as

$$
\begin{aligned}
\tilde{\zeta}= & (1 \text {-average probability of false alarm }) \cdot \operatorname{Pr}\left(H_{0}\right), \\
& +(1 \text {-average probability of detection }) \cdot \operatorname{Pr}\left(H_{1}\right)
\end{aligned}
$$

where $\operatorname{Pr}(\cdot)$ is the occurrence probability of the hypothesis, $H_{0}$ is that the channel is unoccupied by PUs, and

Table 1 Experimental data of the coefficients, $\rho=0.2, \gamma_{k}=9 \mathrm{~dB}$

\begin{tabular}{lllllllll}
\hline$\delta$ & 0.2 & 0.3 & 0.4 & 0.5 & 0.6 & 0.7 & 0.8 & 0.9 \\
\hline$a_{1}$ & 0.96 & 1.02 & 1.03 & 1.02 & 1.01 & 1.01 & 1.01 & 1.00 \\
$a_{0}$ & 10.93 & 4.06 & 1.53 & 1.05 & 0.83 & 0.71 & 0.61 & 0.57 \\
$\beta_{1}$ & 11.56 & 5.73 & 2.95 & 2.29 & 1.96 & 1.72 & 1.59 & 1.52 \\
$\beta_{0}$ & 48.79 & 17.42 & 5.29 & 3.48 & 2.65 & 2.30 & 1.97 & 1.80 \\
\hline
\end{tabular}


Table 2 Fitting results of the coefficients, $\rho=0.2, \gamma_{k}=9 \mathrm{~dB}$

\begin{tabular}{lcclc}
\hline & $\mathrm{a}$ & $\mathrm{b}$ & $\mathrm{c}$ & $\mathrm{d}$ \\
\hline$a_{0}$ & 89.04 & -10.59 & 0.461 & 0.277 \\
$\beta_{1}$ & 63.67 & -9.23 & 1.73 & -0.12 \\
$\beta_{0}$ & 427.7 & -10.81 & 0.77 & 1.01 \\
\hline
\end{tabular}

$H_{1}$ is not. In practice, $\tilde{\zeta}$ can be approximated by the first part of Eq. (8), since the second part is generally much smaller. Based on this, we define $\zeta$ as $\tilde{\zeta} / \operatorname{Pr}\left(H_{0}\right)$ and the energy efficiency as $\eta=\zeta /$ (energy consumed). When the compressed sensing module $\mathbf{A}_{\mathbf{1}}$ is valid, to enhance the spectrum access opportunity, we formulate the problem of energy efficiency maximization as follows.

$$
\begin{aligned}
& \max _{\delta} \eta=\frac{\mathbf{E}\left(\sum_{k=1}^{K}\left(1-P_{f}^{(k)}\right)\right)}{E\left(f_{\mathrm{cs}}, \tau_{s}\right)}, \\
& \text { s.t. } \quad P_{d}^{(k)} \geq \bar{P}_{d}, \quad \forall k, \\
& P_{f}^{(k)} \leq \bar{P}_{f}, \quad \forall k, \\
& \rho<\delta<1
\end{aligned}
$$

The constraint (10) ensures that the primary transmission is not interrupted by the maximization process. The constraints (11) and (12) give an exact range of the compression rate.

To solve the above problem, we firstly focus on the sub-problem as

$$
\max _{\delta} \eta_{k}=\frac{1-P_{f}^{(k)}}{E\left(f_{\mathrm{cs}}, \tau_{s}\right)}, \quad \forall k
$$

Substitute Eqs. (1) and (5) into Eq. (13). Then, the derivation of $\eta_{k}$ against $\delta$ is

$$
\eta_{k}^{\prime}=(\overbrace{E \cdot \exp \left(-v^{2} / 2\right) \cdot v^{\prime} \cdot(1 / \sqrt{2 \pi})}^{\mathrm{A}}-\overbrace{E^{\prime} \cdot(1-Q(v))}^{\mathrm{B}}) / E^{2},
$$

where $v=\left(\beta_{1} / \beta_{0}\right) \sqrt{2 \gamma_{k}+1} Q^{-1}\left(\bar{P}_{d}\right)+\left(\left(\alpha_{1}\left(\gamma_{k}+1\right)-\right.\right.$ $\left.\left.\alpha_{0}\right) \sqrt{M}\right) / \sqrt{2} \beta_{0}$. After some simplifications, e.g., $\alpha_{1} \rightarrow 1$, $\alpha_{0}$, and $\beta_{i} \rightarrow a \cdot \exp (b \cdot \delta), v$ can be approximated as

$$
\begin{aligned}
& v \approx \frac{a_{\beta_{1}}}{a_{\beta_{0}}} \sqrt{2 \gamma_{k}+1} Q^{-1}\left(\bar{P}_{d}\right) \exp \left(\left(b_{\beta_{1}}-b_{\beta_{0}}\right) \delta\right) \\
& \quad+\left(\frac{\gamma_{k}+1}{\sqrt{2} a_{\beta_{0}}} \exp \left(-b_{\beta_{0}} \delta\right)-\frac{a_{\alpha_{0}}}{\sqrt{2} a_{\beta_{0}}} \exp \left(\left(b_{\alpha_{0}}-b_{\beta_{0}}\right) \delta\right)\right) \sqrt{M} .
\end{aligned}
$$

From Table 2, we see that there are small differences between any two $b$ values. When $\delta \in(0,1)$, we have

$$
\begin{aligned}
& v \approx \frac{a_{\beta_{1}}}{a_{\beta_{0}}} \sqrt{2 \gamma_{k}+1} Q^{-1}\left(\bar{P}_{d}\right) \\
& +\left(\frac{\gamma_{k}+1}{\sqrt{2} a_{\beta_{0}}} \exp \left(-b_{\beta_{0}} \delta\right)-\frac{a_{\alpha_{0}}}{\sqrt{2} a_{\beta_{0}}}\right) \sqrt{M}
\end{aligned}
$$

Correspondingly,

$$
v^{\prime}=\frac{-b_{\beta_{0}}\left(\gamma_{k}+1\right)}{\sqrt{2} a_{\beta_{0}}} \exp \left(-b_{\beta_{0}} \delta\right) \sqrt{M}
$$

thus, $v^{\prime}>0$, then $v$ is monotonically increasing as $\delta$ increases. Given the fact that $Q(x)$ is a decreasing function and upper bounded by 1 , we find that both part $\mathrm{A}$ and B of Eq. (14) have non-negative values. Moreover, A and $\mathrm{B}$ are monotonically decreasing and increasing as $\boldsymbol{T}_{\mathbf{k}}=\left\{\hat{T}_{k}^{(0)}, \hat{T}_{k}^{(-1)}, \ldots-\hat{T}_{k}^{(-S+1)}\right\}$ increases, respectively, so $\eta_{k}^{\prime}$ is monotonically decreasing as $\delta$ increases. In the limit, when $\delta \rightarrow \rho$ and $\rho \rightarrow 0$,

$$
\begin{aligned}
& \lim _{\delta \rightarrow \rho, \rho \rightarrow 0} A \approx \frac{-b_{\beta_{0}}\left(\gamma_{k}+1\right)}{2 \sqrt{\pi} a_{\beta_{0}}} \sqrt{M} \cdot E\left(0, \tau_{s}\right)>0, \\
& \lim _{\delta \rightarrow \rho, \rho \rightarrow 0} B \approx 0,
\end{aligned}
$$

then we have $\lim _{\delta \rightarrow \rho, \rho \rightarrow 0} \eta_{k}^{\prime}>0$. On the other hand, when $\delta \rightarrow 1, v$ is close to be a constant being equal to $\sqrt{2 \gamma_{k}+1} Q^{-1}\left(\bar{P}_{d}\right)+\sqrt{M / 2}\left(\gamma_{k}+1\right) ;$ thus, $\lim _{\delta \rightarrow 1} v^{\prime} \approx 0$. Moreover, there is $v>0$ when $\bar{P}_{d} \geq 0.5$. Then, we have $\lim _{\delta \rightarrow 1} A \approx 0$ and $\lim _{\delta \rightarrow 1} B \approx E^{\prime}>0$; thus, $\lim _{\delta \rightarrow 1} \eta_{\mathrm{k}}^{\prime}<0$. As a result, there is a zero point of $\eta_{k}^{\prime}$ within the interval of $\delta \in(0,1)$, so the convexity of $\eta_{k}$ is proved. Considering all the $K$ independent sub-bands, the maximum $\eta$ must also be unique within the range of $\delta$, and it can be easily found through the one-dimensional exhaustive search. In practical application, we can replace the module $\mathbf{A}_{\mathbf{3}}$ by an optimal compression rate matrix indexed by the sparsity order $\rho$ and the SNR vector $\gamma$, which can be stored in the computer's memory. When the estimation of $\rho$ and $\gamma$ is finished, the compression rate $\delta$ can be chosen by searching through the matrix. This makes the energy consumed by $\mathbf{A}_{\mathbf{3}}$ be neglected compared with that of ADC. 


\subsection{SNR and sparsity order estimation $\left(B_{1}\right)$}

To take into account the applicability of the scheme, we give a possible solution of joint SNR and sparsity order estimation in this section. It employs the SNR classification through the multiple-hypothesis-testingbased type-I testing method (see the details in Section $\mathrm{V}$ in [14]). In our applications, the received SNR is partitioned into a noise level plus $k$ signal levels with the strength of each level equal to $(l-1) \mathrm{dB}, l=\{1,2, \ldots, L\}$. Denote the confidence level as $\varepsilon$ and the probability matrix as $\boldsymbol{p}=\left[p_{k, l}\right]_{K \times L}$, where $p_{k, l}$ is the probability that the hypothesis $H_{k, l}$ is accepted, i.e., $\gamma_{k}$ should be classified into signal level $l$. To improve the exactness of SNR classification, $p_{k, l}$ is calculated by using the summary statistic sequence $\boldsymbol{T}_{\mathbf{k}}=\left\{\hat{T}_{k}^{(0)}, \hat{T}_{k}^{(-1)}, \ldots, \hat{T}_{k}^{(-S+1)}\right\}$ obtained from the past $S$ tests before the current moment. The process of SNR classification is as follows:

- Initialization: Let $l=1$ and the set of accepted $H_{k, l}$ for sub-band $k$ as $\boldsymbol{H}_{k}=\left\{H_{k, 1}, H_{k, 2}, \cdots, H_{k, L}\right\}$.

- Step 1: At the signal level $l$, sort $\boldsymbol{p}(:, l)$ in ascending order of $p$ values, $p^{(1)} \leq p^{(2)} \leq \cdots \leq p^{(K)}$, where $\boldsymbol{p}(:, l)$ represents all the elements of the $l$ th column of $\boldsymbol{p}$.

- Step 2: Find the maximum index of $p^{(j)}$ satisfying the condition that $p^{(j)} \leq \frac{j}{L} \varepsilon, j \in\{1,2, \cdots, K\}$, and denote it as $J$.

- Step 3: Reject all the $H_{k, l}$ if $k \in[1, J]$, then eliminate them from $\boldsymbol{H}_{k}$.

- Step 4: If $l=L$, break the loop. Otherwise, $l=l+1$, then go to step 1 .

- Step 5: If $\boldsymbol{H}_{k} \neq \varnothing$, choose the maximum $l$ from $\boldsymbol{H}_{k}$ as the SNR level of sub-band $k$. Otherwise, classify it into the noise level.

Considering all the $K$ sub-bands, the estimation of $\gamma$ can be obtained. Furthermore, after the SNR classification, $\rho$ can be estimated by the number of SNR classified into signal levels

\subsection{Compressed sampling decision $\left(C_{1}\right)$}

Since there is no uniform criterion for judging the sparsity [10], in our application, we say the spectrum is truly sparse if $\rho<0.5$. Finally, by canceling out the threshold variable $\lambda_{k}$ of Eq. (3) when $i=0$ and $i=1$, we evaluate the lower limit of the received SNR $\bar{\gamma}$ with the solution of

$$
M=2 \cdot\left(\frac{\beta_{0} Q^{-1}\left(\bar{P}_{f}\right)-\beta_{1} \sqrt{2 \bar{\gamma}+1} Q^{-1}\left(\bar{P}_{d}\right)}{\alpha_{1}(\bar{\gamma}+1)-\alpha_{0}}\right)^{2},
$$

when $\rho=0.5$ and $\delta=1$, to ensure $M$ is not less than the minimum number of required samples to achieve the pair of target probabilities $\left(\bar{P}_{d}, \bar{P}_{f}\right)$.

\section{Performance analysis and simulations}

In this section, the performance of energy efficiency maximization and energy saving is demonstrated by simulations. We consider a sparse wideband spectrum contains $10 \mathrm{TV}$ bands each with $6-\mathrm{MHz}$ bandwidth. Assume the occurrence probabilities as $\operatorname{Pr}\left(H_{1}\right)=q$, $\operatorname{Pr}$ $\left(H_{0}\right)=1-q$, and $q \in(0,1)$, the number of signal levels as $L=10$, and the received SNRs of the primary signal on each sub-band as $\gamma=\{0,1, \ldots, L-1\}(\mathrm{dB})$. Moreover, assume that $\bar{P}_{d}=0.9, \bar{P}_{f}=0.1, N=512, \varepsilon=0.95, S=20$, and $p_{0}=-95.2 \mathrm{dBm}$.

From Fig. 4, we observe that there is a maximum energy efficiency in each case of $(\rho, \boldsymbol{\kappa})$, where $\boldsymbol{\kappa}$ is the set of occupied sub-bands. As the number of occupied subbands decreases, the optimal compression rate can be reduced, which means a lower sampling ratio is needed. As a result, the maximum energy efficiency can also be substantially improved. For instance, when $\boldsymbol{\kappa}=\{3\}$, the maximum energy efficiency is 728 at the optimal compression rate $\delta=0.55$, then drops to 607 at $\delta=0.8$ when $\boldsymbol{\kappa}=\{3,5,7,9\}$. In addition, all the simulated results match to the numerical results very well, which confirms that the analytical model of the proposed scheme and the process of energy efficiency maximization are valid.

For thorough analysis of energy efficiency maximization, Fig. 5a, b illustrates the maximum energy efficiency and the optimal compression rate, respectively, versus the received SNR and the sparsity order. When focusing on the sparsity orders, $\forall \rho \in\{0.1,02,0.3,0.4\}$, we observe that the higher the received SNR, the larger the maximum energy efficiency can be achieved, and a lower optimal compression rate is needed. Moreover, the positive effect of sparsity order on the energy efficiency can be clearly seen, i.e., the sparser the spectrum, the more energy efficient the proposed scheme.

Finally, to highlight the energy efficiency of our proposed scheme, we compare the energy consumption of the proposal with those of the two sensing schemes mentioned in the introduction, (i) the sequential sensing scheme sampling at the Nyquist rate [3] and (ii) the one-step compressed sensing scheme sampling at the sub-Nyquist rate but aiming at signal reconstruction [5]. Sequentially, denote the energy consumption of the three schemes as $E_{-}$pro, $E_{-}$seq, and $E_{-}$com. In order to facilitate the analysis, under the same sensing performance constraints $\left(\bar{P}_{d} \geq 0.9, \bar{P}_{f} \leq 0.1\right)$, we normalized $E_{-}$pro and $E_{-}$com by $E_{-}$seq, respectively, i.e., $\tilde{E}_{-}$pro $=E_{-}$pro/ $E_{-}$seq, $\tilde{E}_{-}$com $=E_{-}$com $/ E_{-}$seq. In Fig. 6 , we assume that the SNR vector and sparsity order are a priori information and calculate the optimal compression rate through $\mathbf{A}_{3}$. Then, we can obtain the numerical result of $\tilde{E}_{\text {_pro. }}$ On the other hand, we estimate the practical SNR vector and sparsity order through $\mathbf{B}_{\mathbf{1}}$ (in particular, 


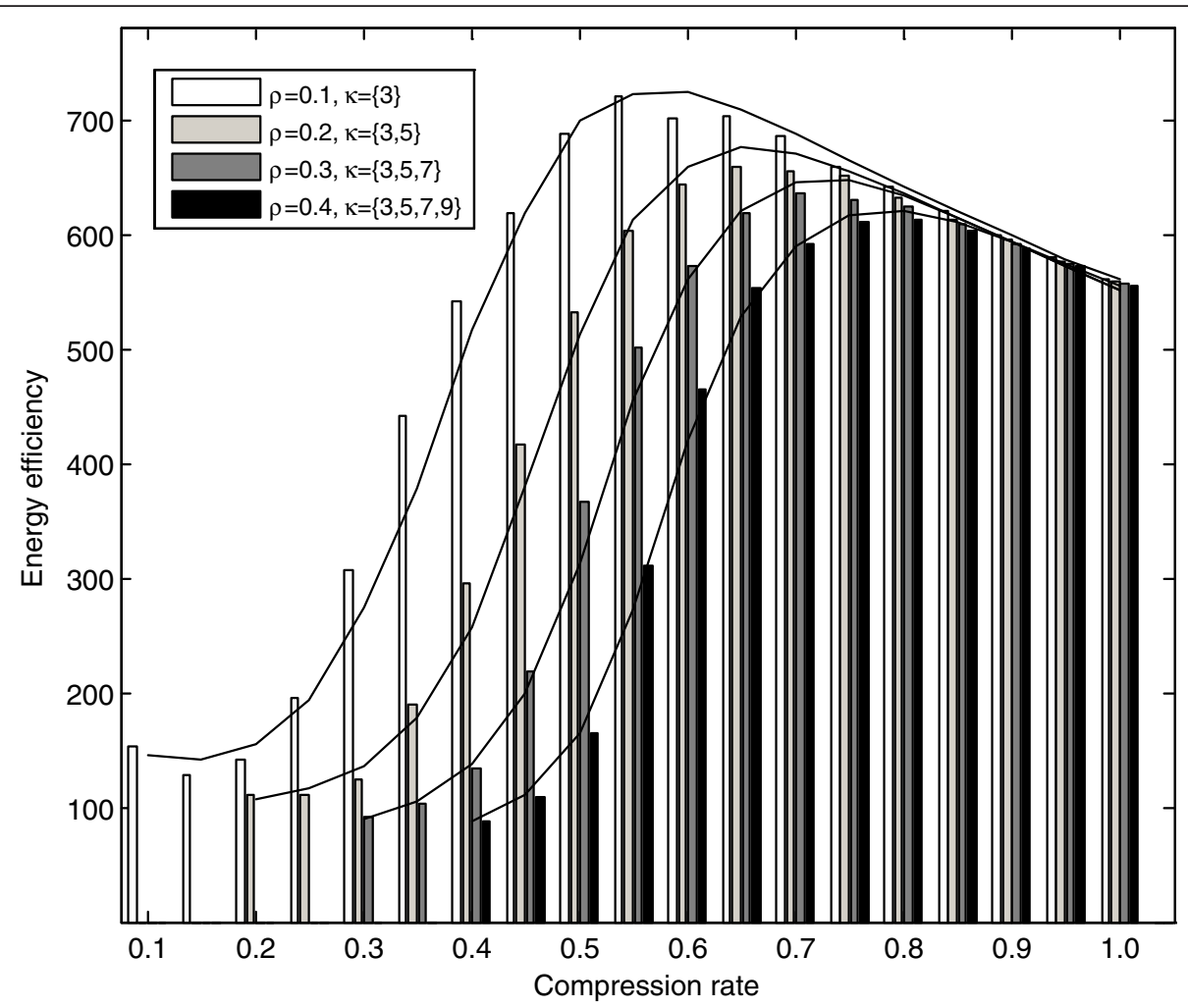

Fig. 4 Energy efficiency (bar for simulated results, line for numerical results)
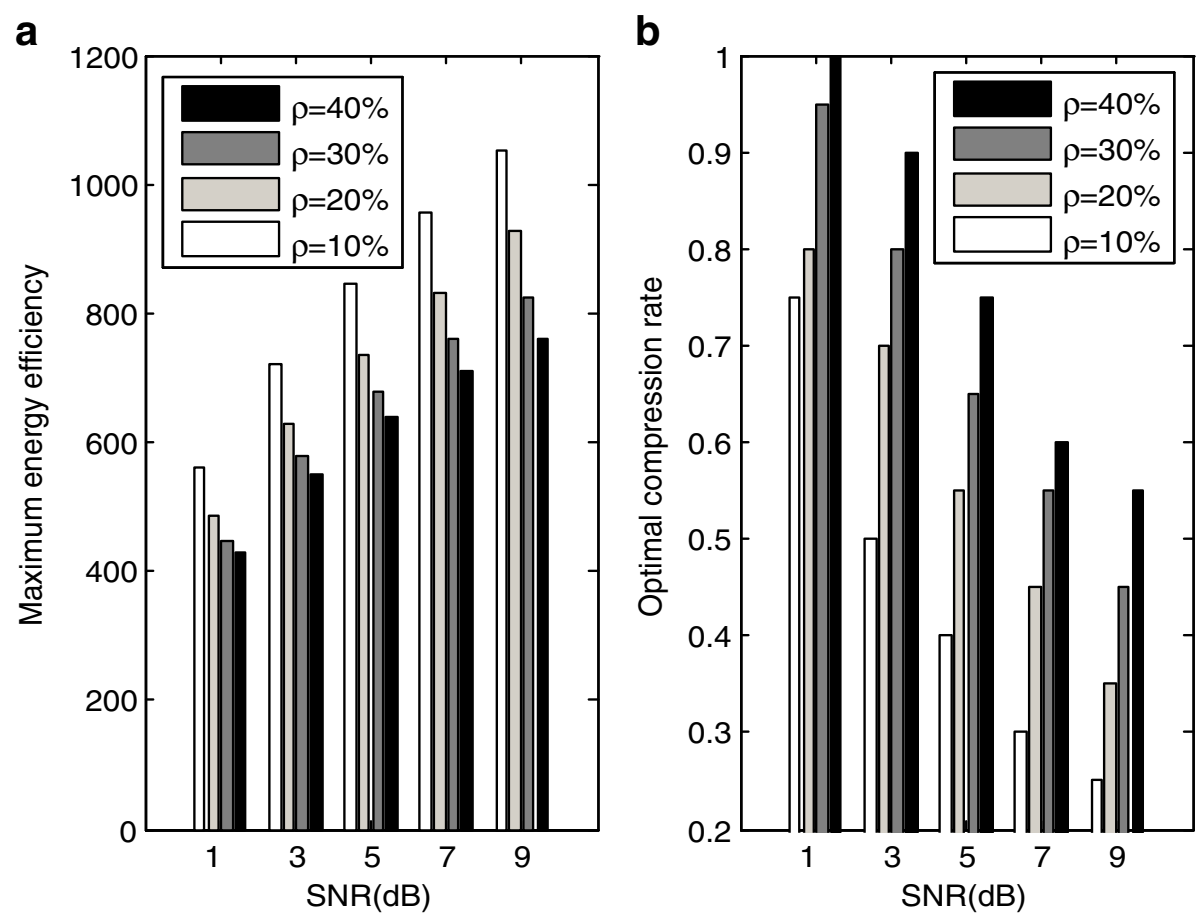

Fig. 5 The maximum energy efficiency and the optimal compression rate versus the received SNR 


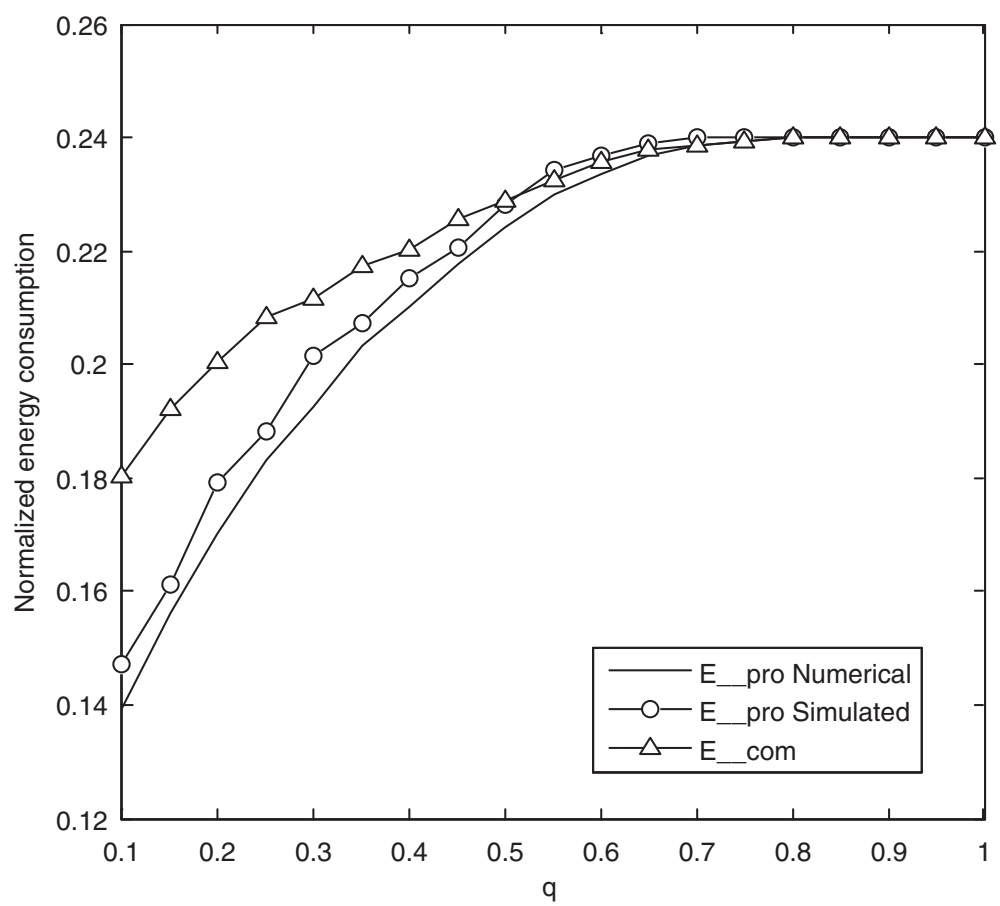

Fig. 6 Normalized energy consumption

to improve the performance of parameter estimation, the signal is sampled at the Nyquist rate in the first 20 sensing time durations) and use them to choose the optimal compression rate from the stored compression rate matrix mentioned above, to obtain the simulated result of $\tilde{E}_{-}$pro. In addition, when performing the one-step compressed sensing scheme as comparison, we use the OMP algorithm to solve the $\ell_{1}$-optimization problem (see the details in formula (11) in [5]). We can see from this figure, the energy consumption of our proposal, $\tilde{E}_{-}$com, is always smaller than that of the one-step compressed sensing scheme, $\tilde{E}_{-}$com. Furthermore, the energy saving is more obvious if $q$ is smaller, which means the wideband spectrum is sparser. However, when $q \geq 0.7$, although $\mathbf{C}_{\mathbf{1}}$ cannot be satisfied in most cases and the sampling rate is chosen to be the Nyquist rate, the energy consumption of the proposal is only about $24 \%$ of that of the sequential sensing scheme, which can be attributed to the parallel multi-band detection scheme used in $\mathbf{A}_{\mathbf{2}}$ shortening the sampling time.

\section{Conclusions}

Taking advantage of the frequency-domain sparsity of the wideband spectrum, a new wideband spectrum sensing scheme has been proposed to save energy through reducing both the sampling rate and sensing time duration. The sparsity order, the received signal-to-noise ratio, and the compression rate are identified as three key factors that affect the sensing performance. Moreover, under the sensing performance constraints, the uniqueness of the maximum energy efficiency within the range of the compression rate has been proved. It is indicated that the sparser the wideband spectrum, the higher the energy efficiency and the lower the energy consumption. All these advantages have been corroborated by simulations.

\section{Competing interests}

The authors declare that they have no competing interests.

\section{Author details}

${ }^{1}$ School of Electronic Information Engineering, Beihang University, Beijing 100191, China. ${ }^{2}$ Beijing Institute of Remote Sensing Equipment, Beijing 100039, China.

Received: 16 April 2015 Accepted: 3 March 2016

Published online: 15 March 2016

\section{References}

1. YY Pei, YC Liang, KC Teh, KH Li, Energy-efficient design of sequential channel sensing in cognitive radio networks: optimal sensing strategy, power allocation, and sensing order. IEEE J. Sel. Areas Commun. 29(8), 1648-1659 (2011)

2. J Meng, W Yin, HH Li, E Hossain, Z Han, Collaborative spectrum sensing from sparse observations in cognitive radio networks. IEEE J. Sel. Areas Commun. 29(2), 327-337 (2011)

3. $\mathrm{M} \mathrm{Xu}, \mathrm{H} \mathrm{Li}, \mathrm{XY}$ Gan, Energy efficient sequential sensing for wideband multi-channel cognitive network, in Proc. IEEE ICC (2011)

4. Z Quan, SG Cui, AH Sayed, HV Poor, Optimal multiband joint detection for spectrum sensing in cognitive radio network. IEEE Trans. Signal Process. 57(3), 1128-1140 (2009) 
5. Z Tian, GB Giannakis, Compressed wideband sensing in cooperative cognitive radio network, in Proc. IEEE GLOBECOM, 2008, pp. 1-5

6. FZ Zeng, C Li, Z Tian, Distributed compressive spectrum sensing in cooperative multihop cognitive networks. IEEE J. Sel. Topics Signal Process. 5(1), 37-48 (2011)

7. YL Polo, Y Wang, A Pandharipande, G Leus, Compressive wideband spectrum sensing, in Proc. IEEE ICASSP, 2009, pp. 2337-2340

8. DD Ariananda, G Leus, Compressive wideband power spectrum estimation. IEEE Trans. Signal Process. 60, 4755-4789 (2012)

9. D Cohen, YC Eldar, Sub-Nyquist sampling for power spectrum sensing in cognitive radios: a unified approach. IEEE Trans. Signal Process. 62(15), 3897-3910 (2014)

10. Y Wang, Z Tian, CY Feng, A tow-step compressed spectrum sensing scheme for wideband cognitive radios, in Proc. IEEE Globecom, 2010

11. Y Wang, Z Tian, CY Feng, Sparsity order estimation and its application in compressive spectrum sensing for cognitive radios. IEEE Trans. Wireless Commun. 11(6), 2116-2125 (2012)

12. AD9257 datasheet, [Online], Available. http://www.analog.com/media/en/ technical-documentation/data-sheets/AD9257.pdf.

13. EJ Candes, MB Wakin, An introduction to compressive sampling. IEEE Personal Commun. 6(4), 12-18 (2008)

14. $\mathrm{SH} \mathrm{Wu}, \mathrm{CY}$ Yang, DHT Huang, Cooperative sensing of wideband cognitive radio: a multiple-hypothesis-testing approach. IEEE Trans. Veh. Technol. 59(4), 1835-1846 (2009)

\section{Submit your manuscript to a SpringerOpen ${ }^{\odot}$ journal and benefit from:}

- Convenient online submission

- Rigorous peer review

- Immediate publication on acceptance

- Open access: articles freely available online

- High visibility within the field

- Retaining the copyright to your article

Submit your next manuscript at $\boldsymbol{s p r i n g e r o p e n . c o m ~}$ 